\title{
In vitro effects of arylimidamides against Besnoitia besnoiti infection in Vero cells
}

\author{
H. C. E. CORTES ${ }^{1}$, N. MULLER ${ }^{2}$, D. BOYKIN ${ }^{3}$, C. E. STEPHENS ${ }^{4}$ and A. HEMPHILL ${ }^{2} *$ \\ ${ }^{1}$ Laboratório de Parasitologia Victor Caeiro, Núcleo da Mitra, ICAAM, Universidade de Évora, Apartado 94, \\ 7000-554 Évora, Portugal \\ ${ }^{2}$ Institute of Parasitology, University of Berne, Längass-Strasse 122, CH-3012 Berne, Switzerland \\ ${ }^{3}$ Department of Chemistry, Georgia State University, PO Box 4098, Atlanta, Georgia 30302-4098, USA \\ ${ }^{4}$ Department of Chemistry and Physics, Augusta State University, Augusta, Georgia 30904-2200, USA
}

(Received 3 November 2010; revised 23 December 2010; accepted 11 Fanuary 2011; first published online 24 February 2011)

\section{SUMMARY}

The in vitro effects of 4 arylimidamides (DB811, DB786, DB750 and DB766) against the proliferative tachyzoite stage of the apicomplexan parasite Besnoitia besnoiti were investigated. These four compounds had been shown earlier to exhibit in vitro activities in the nanomolar range against the related apicomplexans Neospora caninum and Toxoplasma gondii. Real-time-PCR was used to assess $B$. besnoiti intracellular proliferation in vitro. Preliminary assessment by light microscopy identified DB811 and DB750 as the most promising compounds, while DB786 and DB766 were much less effective. Three-day-growth assays and quantitative real-time PCR was used for $\mathrm{IC}_{50}$ determination of DB811 $(0 \cdot 079 \mu \mathrm{M})$ and DB750 $(0.56 \mu \mathrm{M})$. Complete growth inhibition was observed at $1.6 \mu \mathrm{M}$ for DB 811 and $1.7 \mu \mathrm{M}$ for DB750. However, when infected cultures were treated for 14 days, proliferation of parasites occurred again in cultures treated with DB750 from day 4 onwards, while the proliferation of DB811-treated tachyzoites remained inhibited. Electron microscopy of B. besnoitiinfected fibroblast cultures fixed and processed at different time-points following the initiation of drug treatments revealed that DB811 exerted a much higher degree of ultrastructural alterations compared to DB750. These results show that arylimidamides such as DB811 could potentially become an important addition to the anti-parasitic arsenal for food animal production, especially in cattle.

Key words: arylimidamide, Besnoitia besnoiti, in vitro drug treatment, besnoitiosis.

\section{INTRODUCTION}

Besnoitia besnoiti is the causative agent of bovine besnoitiosis, a parasitic disease of cattle widely distributed in sub-Saharian areas, which has recently spread in European countries. Besides Portugal (Cortes et al. 2004), Spain (Juste et al. 1990; Fernandez-Garcia et al. 2010) and France (Jacquiet et al. 2010), bovine besnoitiosis was recently reported in Germany (Schares et al. 2009) and Italy (Gollnick et al. 2010), and it is just a matter of time for the disease to spread to other countries in Europe. Bovine besnoitiosis may have 2 clinical phases. During the initial phase that takes place during the first 4 to 12 days of infection, a high parasitaemia occurs, and the animals present mainly hyperthermia and severe respiratory disorders. Subsequently, in the chronic stage that can last for several years, the parasite shows a high tropism to the skin, leading to dramatic thickening, hardening and folding or wrinkling of the skin, especially around the neck, shoulders and rump, always accompanied by hyperkeratosis,

* Corresponding author: Institute of Parasitology, University of Berne, Längass-Strasse 122, CH-3012 Berne, Switzerland. Tel: +41316312474. Fax: +4131631 2477. E-mail: hemphill@ipa.unibe hyper-pigmentation and alopecia (Pols, 1960). Furthermore, abortion may occur during the acute phase (Juste et al. 1990) and irreversible aspermy is a common sequel that develops during the chronic phase (Cortes et al. 2003, 2005). The above mentioned, together with the refractoriness to all antiparasitic compounds tested so far, render bovine besnoitiosis an important re-emerging disease in the South of Portugal (Cortes et al. 2003).

Despite considerable efforts to identify and develop effective agents to treat bovine besnoitiosis (Shkap et al. 1987), none has yet demonstrated any effect for treatment or prevention of disease. A wide range of anti-infective drugs, including lasalocid, monensin, pirithrexime, pyrimethamine, clindamycin, robenidine and trimethoprim, have previously been shown to exhibit proliferation-inhibitory in vitro activity against closely related Neospora caninum tachyzoites (Lindsay and Dubey, 1989; Lindsay et al. 1994). More recently, artemisinin (Kim et al. 2002), depudecin (Kwon et al. 2003), toltrazuril, ponazuril (Gondim, 2006), nitro-and bromo-thiazolides (Esposito et al. 2005, 2007a,b) and alcoholic herbal extracts (Youn et al. 2004) were reported to be active against $N$. caninum tachyzoites in cell culture. For the treatment of besnoitiosis, several 
commercially available compounds such as oxytetracyclin, sulfonamides, trimethoprim, halofunginone, diaminazene aceturate and pentamidine) were used in gerbils (Shkap et al. 1987). In addition, oxytetracyclin was assessed in $B$. besnoiti-infected rabbits (Shkap et al. 1985). Of these compounds, only oxytetracyclin prevented death in gerbils and rabbits, but only if administered at the moment of infection. More recently, the nitro-thiazolide nitazoxanide and a range of bromo-derivatives were shown to inhibit B. besnoiti tachyzoite proliferation in Vero cells at 5 and $10 \mu \mathrm{g} / \mathrm{ml}$, but these compounds did not affect host cell invasion (Cortes et al. 2007a). Elsheikha and Mansfield (2004) demonstrated the activity of sulfadiazine against $B$. darlingi tachyzoites cultured in bovine turbinate cells.

Arylimidamides represent a class of broadspectrum antimicrobial compounds, which are originally derived from pentamidine. Pentamidine and its analogues exhibit activity against a wide range of intracellular and extracellular protozoan parasites (Bray et al. 2003). Since its discovery, pentamidine has been successfully applied to treat a variety of parasitic infections including African trypanosomiasis, leishmaniasis, and malaria. Diminazene aceturate, a pentamidine-derivative, is commonly used for trypanosome chemotherapy in livestock, but this drug is prone to resistance formation. Other analogues, such as dicationic furans and dicationic carbazole compounds have been reported to exhibit good efficacy against Cryptosporidium parvum in neonatal ICR mouse models (Blagburn et al. 1998a,b). More recently, the development of novel analogues, known as arylimidamides (Wang et al. 2010) lead to derivatives that exhibited a more favourable pharmacokinetic profile, improved bioavailability, lower toxicity, and a higher chance of passing the bloodbrain barrier. We have previously demonstrated that 4 arylimidamides, namely DB750, DB766, DB786, and DB811, exhibited promising activities against $N$. caninum and $T$. gondii tachyzoites in cell culture (Leepin et al. 2008). In this study we describe the in vitro effects of these compounds against $B$. besnoiti tachyzoites grown in human foreskin fibroblasts (HFF) and Vero cells.

\section{MATERIALS AND METHODS}

\section{Culture media, buffers and reagents}

Unless otherwise stated, all tissue-culture media were purchased from Gibco-BRL (Zurich, Switzerland) and biochemical reagents were from Sigma (St Louis, MO). The drugs used in this study were synthesized at the Department of Chemistry and Center for Biotechnology and Drug Design, Georgia State University, USA. They were kept as dry powder or as stock solutions of $1 \mathrm{mg} / \mathrm{ml}$ in dimethyl sulfoxide (DMSO) and were stored at $-20^{\circ} \mathrm{C}$.

\section{Cell culture and parasite purification}

Vero (monkey kidney epithelial) cells were maintained in DMEM supplemented with $2 \%(\mathrm{v} / \mathrm{v})$ inactivated foetal calf serum (FCS), $2 \mathrm{~mm}$ L-glutamine, $50 \mathrm{U}$ of penicillin $/ \mathrm{ml}$, and $50 \mu \mathrm{g}$ of streptomycin $/ \mathrm{ml}$ at $37^{\circ} \mathrm{C}$ with $5 \% \mathrm{CO}_{2}$ in tissue-culture flasks and were trypsinized twice a week. HFF (human foreskin fibroblasts) were maintained in Dulbecco's modified Eagles's medium (DMEM) with $10 \% \mathrm{FCS}, 50 \mathrm{U}$ of penicillin $/ \mathrm{ml}$, and $50 \mu \mathrm{g}$ of streptomycin $/ \mathrm{ml}$ at $37^{\circ} \mathrm{C}$ with $5 \% \mathrm{CO}_{2}$ in tissue-culture flasks. Cultures were trypsinized once a week. B. besnoiti tachyzoites (Bb1Evora03 isolate) were cultured in Vero cell monolayers employing DMEM containing 2\%(v/v) foetal calf serum, $50 \mathrm{IU} / \mathrm{ml}$ penicillin, and $50 \mu \mathrm{g} / \mathrm{ml}$ streptomycin). Parasites were harvested using a cell scraper when they were still intracellular. Harvested material was repeatedly passaged through a 25 -gauge needle at $4{ }^{\circ} \mathrm{C}$ and parasites were purified using cellulose CF11 columns (Cortes et al. 2006).

\section{In vitro drug treatment assays}

In vitro drug treatment assays were carried out in triplicate essentially as previously described for thiazolides (Cortes et al. 2007a). HFF were grown to confluency in 24-well tissue-culture plates, and each well was infected with $5 \times 10^{4}$ cell culturederived and freshly purified $B$. besnoiti tachyzoites in culture medium. Following incubation for $2 \mathrm{~h}$ at $37^{\circ} \mathrm{C}$ in $5 \% \mathrm{CO}_{2}$-atmosphere, unbound parasites were removed by 2 washes in DMEM, and $1 \mathrm{ml}$ of culture medium was added, containing the compounds at concentrations as indicated in the individual experiments. Controls were performed, by adding the solvent (DMSO) at the corresponding concentrations. Subsequently, cultures were maintained at $37^{\circ} \mathrm{C} / 5 \% \mathrm{CO}_{2}$ for different time-periods as indicated below.

A primary evaluation of drug efficacy was performed using light microscopy, by culturing infected cells in the presence of the compounds at concentrations of $2,1,0 \cdot 5,0 \cdot 2$ and $0 \cdot 1 \mu \mathrm{g} / \mathrm{ml}$, for 3 days without medium changes at $37^{\circ} \mathrm{C} / 5 \% \mathrm{CO}_{2}$. The cells were stained with cresyl-violet and efficacies were assessed by counting the numbers of parasites in 10 randomly chosen fields per sample, as previously described (Leepin et al. 2008).

To monitor the direct effects of DB811 and DB750 on HFF and Vero cells, confluent lawns were trypsinized, and cells were resuspended in fresh medium and transferred to 24 -well-plates $\left(5 \times 10^{3}\right.$ cells per well) containing the drugs (at $1.7 \mu \mathrm{M})$ or DMSO as a solvent control. After 2 and $12 \mathrm{~h}$, the adherent cells in 20 different fields were counted by light microscopy. At days 2 and 3, the medium was removed, attached cells were washed with PBS, trypsinized and counted using a Neubauer chamber. For determining the 
effects of DB811 and DB750 on confluent monolayers, cells were transferred to 24-well-plates and grown to $100 \%$ confluency for a period of 6 days. Then the medium was replaced with fresh medium containing DB811 and DB750 (at $1.7 \mu \mathrm{M}$ ) or DMSO, respectively. After 6 days, the medium was removed, attached cells were washed with PBS, trypsinized and counted using a Neubauer chamber.

For the determination of $\mathrm{IC}_{50}$ values for $\mathrm{DB} 811$ and DB750, infected cultures were treated with different drug concentrations, ranging from $0 \cdot 01$ to $3 \cdot 4 \mu \mathrm{M}$, for a period of 3 days. In some assays, the cultivation periods were extended to 14 days. Samples for quantitative real-time PCR analysis were taken by removal of the medium and addition of a mixture of $200 \mu \mathrm{l}$ of phosphate-buffered saline, $180 \mu \mathrm{l}$ of lysis buffer and $20 \mu \mathrm{l}$ of proteinase K (DNAeasy Kit, QIAGEN, Basel, Switzerland). Samples were transferred to an Eppendorf tube and frozen at $-20^{\circ} \mathrm{C}$ until further processing. Following quantitative realtime PCR (see below), $\mathrm{IC}_{50}$ values were calculated after the logit-log-transformation of the relative growth $(\mathrm{RG}$; control $=1)$ according to the formula $\ln \{(\mathrm{RG} /(1-\mathrm{RG})\}=\mathrm{a} \times \ln ($ drug concentration $)+\mathrm{b}$ and subsequent regression analysis by the corresponding software tool contained in the Excel software package (Microsoft, Seattle, WA, USA).

In order to assess the effects of compounds on already established cultures, the drugs were added at $24 \mathrm{~h}$ post-infection, and samples were collected at different time-points and processed for real-time $\mathrm{PCR}$ as indicated below.

\section{Processing of DNA samples and LightCycler-based quantitative PCR}

DNA purification was done with the DNAeasy kit from Qiagen (QIAGEN) according to the protocol for tissue samples. Samples were then eluted in a volume of $100 \mu$ l of AE buffer (QIAGEN) and boiled for $5 \mathrm{~min}$. For $B$. besnoiti, detection of DNA amplification products and quantification of parasite numbers through fluorescence resonance energy transfer were performed on the LightCycler instrument (Roche Diagnostics, Basel, Switzerland) as previously described (Cortes et al. 2007b).

As external standards, samples containing the DNA from 10, 100 and 1000 B. besnoiti tachyzoites were included. The parasite count for a given sample was calculated by interpolation from this standard curve. Each assay in a given experiment was carried out in quadruplicate, and the outcome of 1 representative experiment of at least 3 independent experiments, all producing virtually identical results, is shown.

\section{Transmission electron microscopy}

HFF monolayers were grown to confluency, infected with $B$. besnoiti tachyzoites, and at $24 \mathrm{~h}$ post-infection, treatments with DB750 (1.7 $\mu \mathrm{M})$ and DB811 $(1 \cdot 6 \mu \mathrm{M})$ were initiated. After 24,48 and $72 \mathrm{~h}$, medium was removed, samples were washed

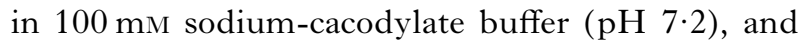
specimens were fixed in cacodylate buffer containing $2 \cdot 5 \%$ glutaraldehyde. After $10 \mathrm{~min}$, cells were scraped off with a rubber policeman, centrifuged for $10 \mathrm{~min}$ at $4^{\circ} \mathrm{C}$ at $1000 \mathrm{~g}$, and the resulting pellet was further fixed at $4{ }^{\circ} \mathrm{C}$ overnight, followed by post-fixation in $1 \% \mathrm{OsO}_{4}$ in cacodylate buffer for $4 \mathrm{~h}$ at $4{ }^{\circ} \mathrm{C}$. Subsequently, specimens were washed in water and were pre-stained in $1 \%$ uranyl acetate in water for $1 \mathrm{~h}$ at $4{ }^{\circ} \mathrm{C}$, followed by extensive washing in water. After dehydration in a graded series of ethanol (30-50-7090-100\%), they were embedded in Epon 820 epoxy resin over a period of 2 days with 3 resin changes. The polymerization of the resin was done at $65^{\circ} \mathrm{C}$ for $24 \mathrm{~h}$. Ultrathin sections were cut on a Reichert and Jung ultramicrotome and were loaded onto 300-mesh copper grids (Plano GmbH, Marburg, Germany). Staining with uranyl acetate and lead citrate was performed as described (Hemphill et al. 2004). Grids were viewed on a Phillips 400 TEM operating at $80 \mathrm{kV}$.

\section{Statistical analysis}

The significance of the differences between end-point values of the control and experimental assays in the growth and inhibition experiments was determined by Student's $t$-test, using the Microsoft Excel program. $P$ values of $<0.05$ were considered statistically significant.

\section{RESULTS}

\section{Effects of arylimidamides on the proliferation of} B. besnoiti tachyzoites in vitro

The 4 arylimidamides previously shown to be active against $N$. caninum and $T$. gondii tachyzoites (Leepin et al. 2008) were evaluated in HFF cultures infected with $B$. besnoiti tachyzoites. Compounds were first assessed light microscopically using 3-day-growth assays, and the drugs were added only once, right after the time-point of infection, at 2, 1, 0.5, $0 \cdot 2$ and $0 \cdot 1 \mu \mathrm{g} / \mathrm{ml}$, respectively. This light-microscopical assessment showed that DB811 and DB750 inhibited $B$. besnoiti proliferation even at the lowest concentration $(0.1 \mu \mathrm{g} / \mathrm{ml})$, while the other two were less active, and concentrations above $0.5 \mu \mathrm{g} / \mathrm{ml}$ were required to detect a proliferation inhibition (see Table 1).

DB811 and DB750 exhibited selective toxicity against $N$. caninum tachyzoites. They did not have any notable effects on HFF or Vero host cell viability and growth when applied at concentrations up to $1.7 \mu \mathrm{M}$. Following trypsinization, drug-treated host cells adhered readily to the plastic surface of tissueculture devices in the presence of both drugs, underwent cell division at a similar rate as untreated 
Table 1. Structures of DB compounds evaluated in this study

(Primary assessment of drug efficacy (growth of B.b) was done at concentrations of 2, $1,0 \cdot 5,0 \cdot 2$ and $0 \cdot 1 \mu \mathrm{g} / \mathrm{ml}$ (corresponding to after 3 days of culture). The evaluation was performed using light microscopy following staining with cresyl-violet. Efficacies were assessed by counting the numbers of parasites in 10 randomly chosen fields per sample. Samples were scored as either "-" (= no visible tachyzoites), “+” (=1-50 tachyzoites), “++” (= 50-500 tachyzoites) and "+++” (= more than 500 tachyzoites). IC $_{50}$ values (assessed by real-time PCR) were only determined for DB750 and DB811; n.d., not done.)

\begin{tabular}{|c|c|c|c|}
\hline Code & Structure & $\begin{array}{l}\text { Growth B.b. } \\
(>0 \cdot 1 \mu \mathrm{g} / \mathrm{ml})\end{array}$ & $\begin{array}{l}\mathrm{IC}_{50} \\
\text { B. besnoiti }\end{array}$ \\
\hline DB75 & & +++ & n.d. \\
\hline DB750 & & - & $0 \cdot 56 \mu \mathrm{M}$ \\
\hline DB766 & & + & n.d. \\
\hline DB786 & & ++ & n.d. \\
\hline DB811 & & - & $0 \cdot 079 \mu \mathrm{M}$ \\
\hline
\end{tabular}

cells, and there was no visibly detectable impairment of confluent monolyers observed over an incubation period of up to 6 days (data not shown).

The $\mathrm{IC}_{50}$ s of DB811 and DB750 were assessed in 3-day-growth assays, by employing real-time PCR for quantifying the numbers of tachyzoites. The $\mathrm{IC}_{50}$ s of the two compounds were $0.079 \mu \mathrm{M}$ for DB811 and $0.56 \mu \mathrm{M}$ for DB750 (Table 1). Further, at a concentration of $1.7 \mu \mathrm{M}$ for DB750 and $1.6 \mu \mathrm{M}$ for DB811, drug treatments resulted in complete inhibition of parasite proliferation. No effects on either Vero cell nor HFF host cell monolayers were observed, confirming the earlier findings reported by Leepin et al. (2008). Thus, both DB811 and DB750 exhibited selective toxicity against $B$. besnoiti at submicromolar levels.

The effects of treatments with DB811 and DB750 on parasite proliferation in $B$. besnoiti-infected cells over a longer period of time (14 days) were also investigated (Fig. 1). Again, the compounds were added only once, shortly following infection. Treatment with DB811 at $1.6 \mu \mathrm{M}$ resulted in consistent repression of parasite proliferation during this 14-day period (Fig. 1A), and light microscopical inspection did not reveal any alterations in the structural integrity of the host cell monolayers. Additional experiments were performed, during which DB811 treatment was carried out for $3 \cdot 5 \mathrm{~h}$ only, followed by removal of the drug through washes with medium. Subsequent culturing in the absence of DB811 showed that a treatment duration as short as $3 \cdot 5 \mathrm{~h}$ was already sufficient to exert an effect that consistently inhibited parasite proliferation (Fig. 1B) In contrast to DB811, DB750 did not maintain its antiparasitic activity for 14 days, and parasite numbers started to increase again after day 4 of treatment (Fig. 1A), indicating that the anti-proliferative activity of this compound was only transient.

We then investigated whether DB750 and DB811 would also be effective if drug treatment was initiated at later stages of infection ( $24 \mathrm{~h}$ post-infection), when parasitophorous vacuoles containing numerous tachyzoites had already formed. Figure 2 demonstrates that DB811, applied at $1.6 \mu \mathrm{M}$ exhibited a massive and immediate inhibitory effect, while DB750 applied at $1.7 \mu \mathrm{M}$ did not result in a consistent repression of tachyzoite proliferation (Fig. 2).

\section{Effects of DB811 and DB750 on the ultrastructure of $\mathrm{B}$. besnoiti tachyzoites}

The morphological and structural alterations associated with DB811- and DB750-treatments were studied by TEM, with specimens that were fixed and processed at different time-points after initiation of treatments. In untreated control cultures (Fig. 3A, B), B. besnoiti tachyzoites were found within a parasitophorous vacuole, surrounded by a parasitophorous vacuole membrane, and proliferating through endodyogeny (Fig. 3A). Parasites actively secreted 

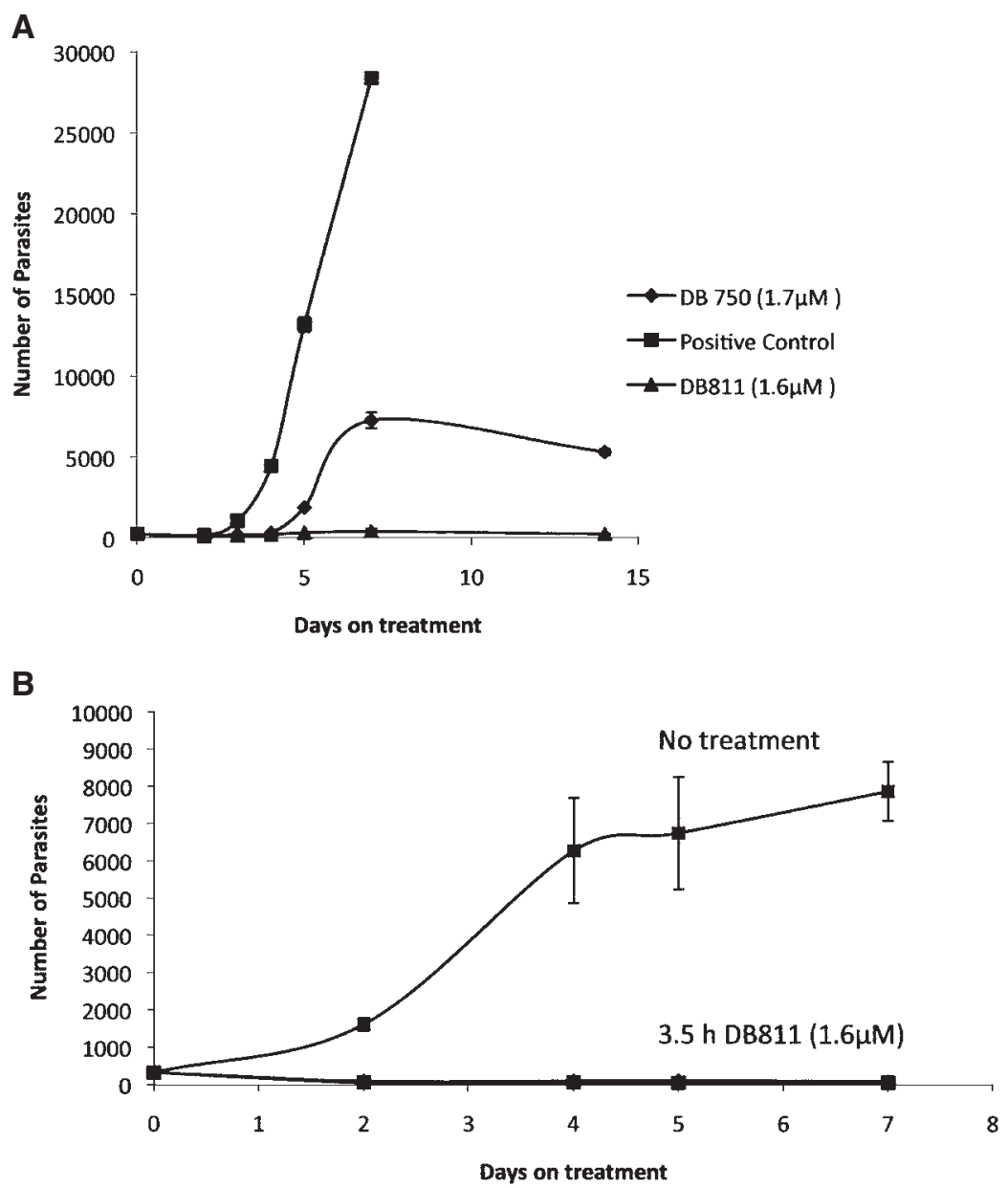

Fig. 1. Effects of DB811 and DB750 on Besnoitia besnoiti proliferation. (A) Proliferation of B. besnoitia tachyzoites in vitro for a period of 14 days as assessed by real-time PCR. Note the initial inhibiton of proliferation by both compounds. On day 5, DB750 lost anti-proliferative properties, while DB811 did not. Positive control= no treatment. (B) Infected Vero cells were incubated with DB811 $(1.6 \mu \mathrm{M})$ for $3.5 \mathrm{~h}$, or were left untreated. Note that the incubation of $B$. besnoiti with $1 \cdot 6 \mu \mathrm{M}$ DB811 had a lasting inhibitory effect. Error bars represent standard deviations.

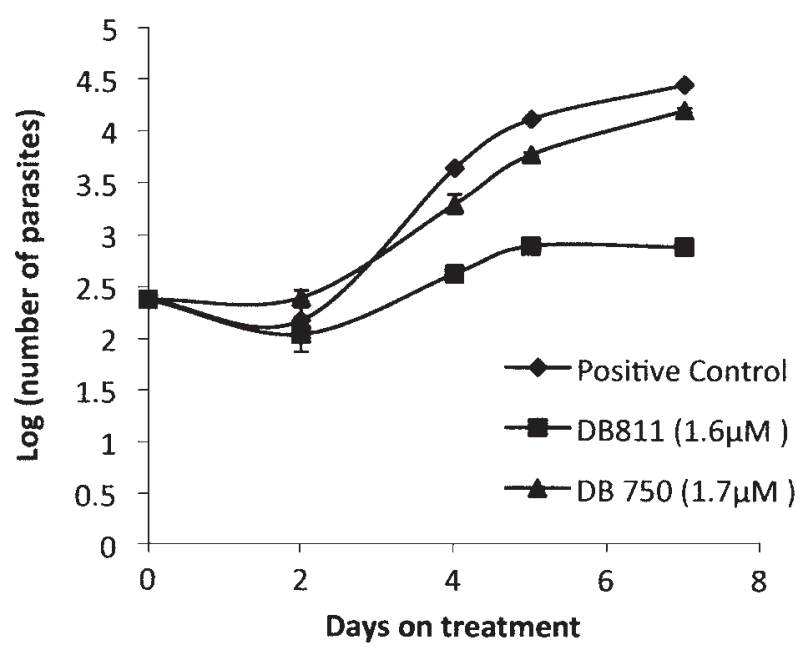

Fig. 2. Effects of DB811 and DB750 on established infections in vitro. Vero cells were infected with Besnoitia besnoiti tachyzoites for $24 \mathrm{~h}$, and were treated by the addition of DB811 $(1.6 \mu \mathrm{M})$ and DB750 $(1.7 \mu \mathrm{M})$. Note that only DB811 had a marked impact on tachyzoite proliferation. Positive control= no treatment. Error bars represent standard deviations. membranous material and possibly other metabolites into the lumen of the vacuole. Tachyzoites exhibited the typical features of apicomplexan parasites such as conoid and micronemes at the apical tip, and rhoptries with an electron-dense and amorphous appearance (Fig. 3A). At later time-points of infection (Fig. 3B), tachyzoites were more densely packed within the vacuole, and embedded into a granular matrix.

In DB750-treated cultures fixed and processed after $24 \mathrm{~h}$, mostly smaller vacuoles could be observed (Fig. 3C), indicating that initially DB750 could have exerted an inhibitory effect. In some instances, tachyzoites exhibiting a completely unaltered ultrastructure were seen side-by-side to vacuoles containing very electron-dense and obviously non-viable parasite residues. However, at later time-points (48-72 h), tachyzoites became more numerous, obviously underwent cell division, and formed larger pseudocysts, as shown in Fig. 3D and E.

In DB811-treated parasites, severe alterations in parasite ultrastructure were visible already after $24 \mathrm{~h}$ (Fig. 4A) and $48 \mathrm{~h}$ (Fig. 4B) of incubation. 

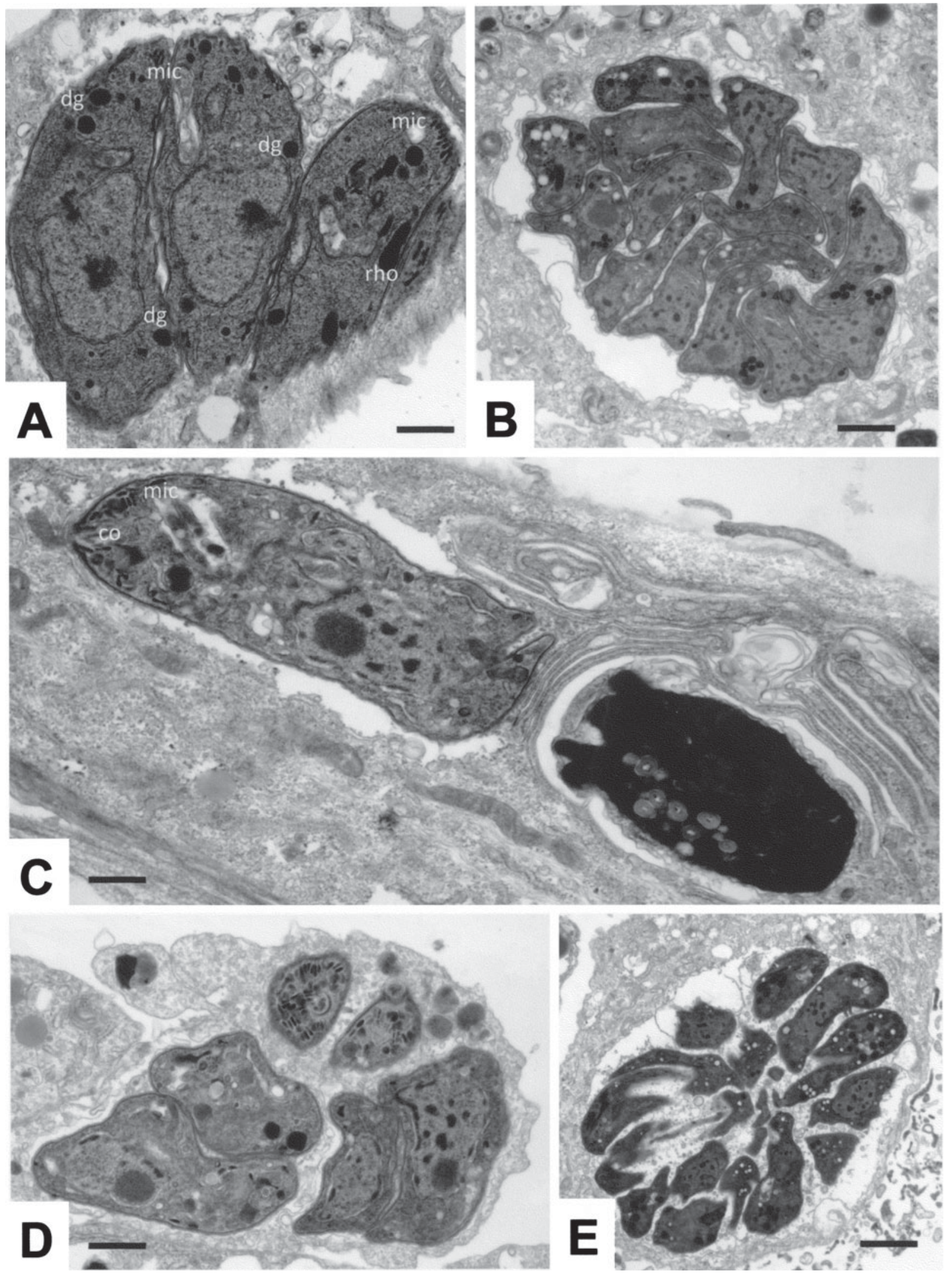

Fig. 3. TEM of HFF infected with Besnoitia besnoiti tachyzoites. (A) and (B) represent untreated controls, fixed and processed at $24 \mathrm{~h}(\mathrm{~A}$; Scale bar $=0.5 \mu \mathrm{m})$ and $72 \mathrm{~h}(\mathrm{~B}$; Scale bar $=1.4 \mu \mathrm{m})$ of infection. $(\mathrm{C})-(\mathrm{E})$ are B. besnoitia that were exposed to $1.7 \mu \mathrm{M}$ DB750 for $24 \mathrm{~h}(\mathrm{C}), 48 \mathrm{~h}(\mathrm{D})$ and $72 \mathrm{~h}(\mathrm{E})$. (C) Shows an unaffected tachyzoite (on the left) and a largely distorted parasite (on the right) within the same cell; Scale bar $=0.5 \mu \mathrm{m}$. Scale bars in (D) $=0 \cdot 8 \mu \mathrm{m} ;(\mathrm{E})=2 \mu \mathrm{m}$. co, conoid; mic, micronemes; rho, rhoptries; dg, dense granules. 


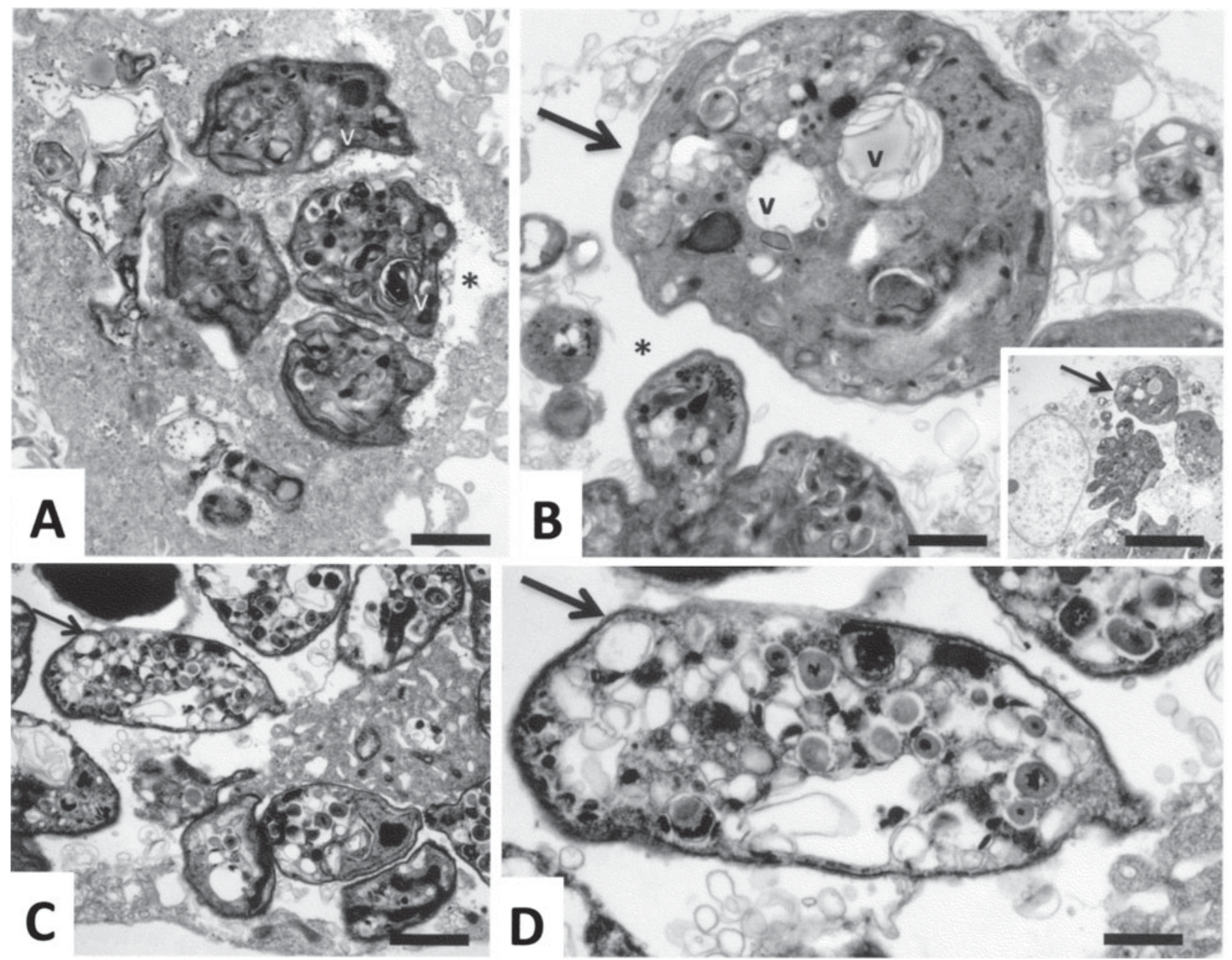

Fig. 4. TEM of Besnoitia besnoitia-infected HFF undergoing DB811-treatment. HFF were infected with B. besnoiti tachyzoites and treated with DB811 (1.6 $\mathrm{M})$, Samples were fixed and processed after $24 \mathrm{~h}(\mathrm{~A}), 48 \mathrm{~h}(\mathrm{~B})$ and $72 \mathrm{~h}(\mathrm{C}, \mathrm{D})$ of incubation. Dramatic alterations of tachyzoite ultrastructure are seen already at $24 \mathrm{~h}$ of drug treatment (A; Scale $\mathrm{bar}=1 \mu \mathrm{m})$ and $(\mathrm{B} ;$ Scale bar $=0.4 \mu \mathrm{m})$. The insert in $(\mathrm{B})$ represents a low magnification overview, and the arrows point towards the same parasite. (D) Represents a higher magnification view of (C), and the arrows point towards the same tachyzoite. At $72 \mathrm{~h}$, alterations become more evident. Scale bar in $(C)=1 \mu \mathrm{m}$; in $(\mathrm{D})=0 \cdot 3 \mu \mathrm{m}$.

Tachyzoites exhibited numerous cytoplasmic vacuoles which were filled with either membranous or electron-dense material, or appeared empty. Parasite plasma membranes and nuclei still maintained their integrity, but the parasitophorous vacuole membrane could no longer be clearly delineated in many instances. In some vacuoles, the granular material constituting the granular matrix components of the parasitophorous vacuole was still visible, while in others the matrix had vanished. At later time-points such as $72 \mathrm{~h}$ post-initiation of treatment (Fig. 4C, D), the damage became more dramatic, with even increased vacuolization. Virtually all parasites exhibited a non-viable morphology and an increasing number of intracellularly located tachyzoite ghosts became visible.

\section{DISCUSSION}

DB750, DB766, DB786 and DB811 are arylimidamide compounds that had been identified earlier to exhibit good activities against the apicomplexan parasites $T$. gondii and $N$. caninum. Thus, the efficacy of these drugs against $B$. besnoiti, which is closely related to Toxoplasma and Neospora, was investigated. Surprisingly, only DB750 and DB811 were shown to substantially inhibit the proliferation of $B$. besnoiti in 3-day assays, with $\mathrm{IC}_{50}$ values of $0.56 \mu \mathrm{M}$ and $0.079 \mu \mathrm{M}$ respectively, while the other 2 compounds did not provide satisfying results. In earlier studies on $N$. caninum, all 4 compounds exhibited $\mathrm{IC}_{50}$ s between $0 \cdot 16$ and $0.66 \mu \mathrm{M}$, thus in a similar range.

These 4 arylimidamides all represent modified versions of DB75 (furamidin), and are built symmetrically, with the 2 core structure-benzene rings being altered through the addition of either 2 chloro atoms (DB811), 2 hydroxy groups (DB750), or iso-propoxy groups (DB786 and DB766). The results of this study suggest that the iso-propoxy-modifications on the 2 core-structure benzene rings had a negative impact on the efficacy of these compounds against 
B. besnoiti tachyzoites. However, other compounds should be investigated in order to properly analyse the structure-activity relationship.

These 4 compounds are related, in that they are derived from furamidine, a bis-amidine diphenylfuran derivative, with hydrophobic moieties substituting the amidine ends of the molecules. However, at concentrations up to $5 \mu \mathrm{g} / \mathrm{ml}$, furamidine itself was ineffective against $N$. caninum, $T$. gondii (Leepin $e t$ al . 2008) and B. besnoiti (data not shown).

$N$-phenyl-substitution of furamidine was shown earlier to markedly increase its anti-parasitic activity against other Trypanosoma cruzi, with $\mathrm{IC}_{50}$ values in the lower micromolar $(2-4 \mu \mathrm{M})$ range (de Souza et al. 2004). In addition, phenyl-substituted furamidine caused apoptosis-like death in T. cruzi (de Souza et al. 2006). Thus, the addition of hydrophobic groups at the two ends of the molecule could increase membrane permeability. Prospectively, this could also increase the bioavailability of these compounds in vivo. This is important for apicomplexan parasites, for which any compound that needs to target these organisms has to cross at least 3 distinct membrane layers: the host cell membrane, the membrane of the parasitophorous vacuole, and the parasite plasmalemma. In addition, depending on the mechanism(s) of action, other organellar membranes (mitochondria, Golgi) could also get involved. At present we do not know how the drugs traverse through these compartments, or how they reach these intracellular parasites. Arylimidamides display $\mathrm{pKa}$ values that are significantly lower compared to those of diamidines such as pentamidine and furamidine (Wang et al. 2010). Therefore, the physicochemical properties of arylimidamides could influence the ability of these compounds to act on intracellular organisms. For instance, DB766, admittedly not very active against $B$. besnoiti tachyzoites (this study), was reported to exhibit excellent in vivo activity in laboratory models for visceral leishmaniasis (Wang et al. 2010) and was shown to be highly efficacious in both in vitro and in vivo laboratory models for T. cruzi infection (Batista et al. 2010). In addition, DB766 has favourable characteristics in terms of oral bioavailability, pharmacokinetics and selective toxicity, rendering this compound, and its class, predestined for further pre-clinical evaluation as an oral treatment for visceral leishmaniasis and Chagas disease (Wang et al. 2010; Batista et al. 2010).

DB750 and DB811 exhibited different $\mathrm{IC}_{50}$ values, and complete inhibition of $B$. besnoiti tachyzoite proliferation was achieved at very similar concentrations of $1.7 \mu \mathrm{M}$ and $1.6 \mu \mathrm{M}$, respectively. However, when drug treatments lasted for longer periods of time, we found that DB750 lost its activity, resulting in tachyzoite proliferation with time, while DB811 did not. There are several possibilities that could account for that. On one hand, it is possible that the two compounds are different in terms of stability in medium, or that they are metabolized differently by the host cells. However, earlier investigations (Leepin et al. 2008) had demonstrated that pretreatment of HFF with DB750 in the absence of $N$. caninum tachyzoites, washing of cells and subsequent infection with $N$. caninum tachyzoites and culturing in the absence of the drug, profoundly impaired $N$. caninum tachyzoite proliferation. Thus, this 'memory-effect' described in $N$. caninuminfected cells relates to internalized, and possibly metabolized drug, and is apparently not relevant in Besnoitia-infected cells. The stability of DB750 in culture medium for extended periods of time has not been investigated so far, but our study indicates that the compound only acts parasitostatically and not parasitocidally and, due to possible instability in the culture medium, the parasite resumes proliferation.

Indeed, we found that incubation of B. besnoitiiinfected cell cultures with DB811 at a concentration of $1.6 \mu \mathrm{M}$ for a period of only $3.5 \mathrm{~h}$ is already sufficient to prevent further growth of tachyzoites for a period of 8 days, demonstrating the parasiticidal activity of the drug. In addition, electron microscopy of DB811-treated B. besnoitii-infected host cells showed clear signs of parasite deterioration already after $24 \mathrm{~h}$ of treatment with DB811, but only very limited effects were seen with DB750. DB811-treated tachyzoites were altered by the presence of increased cytoplasmic vacuolization and electron-dense inclusions, which indicated severe metabolic disturbances, similar to those observed earlier in $B$. besnoitii tachyzoites following in vitro treatment with thiazolides (Cortes et al. 2007a), However, thiazolides are active only at much higher concentrations (approx. $10 \mu \mathrm{g} / \mathrm{ml}$ ), while anti-proliferative effects with DB811 on Besnoitia were visible already concentrations below $0 \cdot 1 \mu \mathrm{g} / \mathrm{ml}$. The presence of the 2 chloro atoms on the core-structure benzene rings of DB811 could have an important impact, leading to much more rapid and efficient killing of $B$. besnoiti tachyzoites. However, the structural differences between DB750 and DB811 involve not only replacement of $\mathrm{OH}$ groups by chloro atoms but also a change in ring position. Consequently, further studies are required to determine if the differences are due to replacement of $\mathrm{OH}$ by $\mathrm{Cl}$ or the change in position of the substitution or a combination of both.

The observed initial inhibition of $B$. besnoiti proliferation, as observed in 3-day-growth assays, was expected, based on earlier in vitro investigations on $N$. caninum and $T$. gondii tachyzoites (Leepin et al. 2008). However, the fact that these parasites resumed with endodyogeny from day 4-5 onwards, was surprising, and indicated that Besnoitia tachyzoites are highly adaptable and have the capacity to react to adverse conditions induced by drug treatment, within a short time frame of a few days. How this is achieved is not known, but as for other protozoan parasites, regulatory pathways that act through 
chromatin structure, thus epigenetic effects, could account for this (reviewed by Bougdour et al. 2010 and Gomez et al. 2010). Indeed, we have recently obtained similar findings in experiments that showed that $T$. gondii tachyzoites rapidly adapted to the action of DB750 in vitro (Christian Kropf, Andrew Hemphill, manuscript in preparation). This clearly shows that these intracellular cyst-forming apicomplexan parasites, although morphologically and phylogenetically closely related, exhibit unique properties. These results also suggest that the $\mathrm{IC}_{50}$ values that are determined in short-term treatment assays, although useful as a measure for anti-parasitic activities, should be treated with caution, and longterm effects must also be studied.

In conclusion, we propose that the arylimidamines should be further investigated for the treatment of besnoitiosis. This is particularly important since there is no drug currently available for the treatment of this disease. Clearly, in chronically diseased cattle the situation is much different, as these animals suffer from severe skin lesions caused by the cyst-forming bradyzoite stage. The situation is even more complicated, as a secondary cyst wall surrounds the multinucleated host skin cell. These structures represent impressive barriers that protect the parasites from immunological and physiological reactions on the part of the host and, most likely also from potentially active pharmaceutical compounds. Further studies should address this question, by developing a skin culture system employing infected skin samples which could, at least in part, mimic the in vivo-situation.

\section{ACKNOWLEDGEMENTS}

This study was supported by a grant provided by the Swiss National Science Foundation (grant no. 31-127374).

\section{REFERENCES}

Batista, D. G. J., Batista, M. M., de Oliveira, G. M., do Amaral, P. B., Lannes-Vieira, J., Britto, C. C., Junqueira, A., Lima, M. M., Romanha, A. J., Sales, P.A.S, Stephend, C.E., Boykin, D.W. and Soeiro, M. N. C. (2010). Arylimidamide DB766, a potential chemotherapeutic candidate for Chagas' disease treatment. Antimicrobial Agents and Chemotherapy 54, 2940-2954.

Blagburn, B. L., Drain, K. L., Land, T. M., Kinard, R. G., Moore, P. H., Lindsay, D.S., Patrick, D.A., Boykin, D.W. and Tidwell, R. R. $(1998 a)$. Comparative efficacy evaluation of dicationic carbazole compounds, nitazoxanide, and paromomycin against Cryptosporidium parvum infections in a neonatal mouse model. Antimicrobial Agents and Chemotherapy 42, 2877-2882.

Blagburn, B. L., Drain, K. L., Land, T. M., Moore, P. H., Kinard, R. G., Lindsay, D. S., Kumar, A., Shi, J., Boykin, D. W. and Tidwell, R. R. (1998b). Dicationic furans inhibit development of Cryptosporidium parvum in HSD/ICR suckling Swiss mice. Fournal of Parasitology 84, 851-856.

Bougdour, A., Braun, L., Cannella, D. and Hakimi, M. A. (2010). Chromatin modifications: implications in the regulation of gene expression in Toxoplasma gondii. Cellular Microbiology 12, 413-423, doi:CMI1446 [pii];10.1111/j.1462-5822.2010.01446.x [doi]

Bray, P. G., Barrett, M. P., Ward, S. A. and De Koning, H. P. (2003). Pentamidine uptake and resistance in pathogenic protozoa: past, present and future. Trends in Parasitology 19, 232-239, doi:S1471492203000692 [pii]. Cortes, H., Ferreira, M. L., Silva, J.F., Vidal, R., Serra, P. and Caeiro, V. (2003). Contribuição para o estudo da besnoitiose bovina em Portugal. Revista Portuguesa de Ciências Veterinárias 98, no. 545, 43-46.
Cortes, H., Leitão, A., Vidal, R., Soares, H., Marques, I., Reis, Y., Waap, E., Pereira da Fonseca, I., Fazendeiro, I., Ferreira, M. L. and Caeiro, V. (2004). Identification, isolation and sequence variability of Besnoitia besnoiti in Portugal. Proceedings of COST Action 857, 1st Annual Workshop, May 3-6th, Lisbon, Portugal.

Cortes, H., Leitão, A., Vidal, R., Vila-Vicosa, M. J., Ferreira, M. L., Caeiro, V. and Hjerpe, C. A. (2005). Besnoitiosis in bulls in Portugal. Veterinary Record 157, 262-264.

Cortes, H. C., Mueller, N., Esposito, M., Leitao, A., Naguleswaran, A. and Hemphill, A. (2007a). In vitro efficacy of nitro- and bromo-thiazolylsalicylamide compounds (thiazolides) against Besnoitia besnoiti infection in Vero cells. Parasitology 1-11.

Cortes, H. C., Reis, Y., Gottstein, B., Hemphill, A., Leitao, A. and Muller, N. (2007b). Application of conventional and real-time fluorescent ITS1 rDNA PCR for detection of Besnoitia besnoiti infections in bovine skin biopsies. Veterinary Parasitology 146, 352-356.

Cortes, H. C., Reis, Y., Waap, H., Vidal, R., Soares, H., Marques, I., Pereira, d. F. I., Fazendeiro, I., Ferreira, M. L., Caeiro, V., Shkap, V., Hemphill, A. and Leitao, A. (2006). Isolation of Besnoitia besnoiti from infected cattle in Portugal 2. Veterinary Parasitology 141, 226-233.

de Souza, E. M., Lansiaux, A., Bailly, C., Wilson, W. D., Hu, Q., Boykin, D. W., Batista, M. M., Araujo-Jorge, T. C. and Soeiro, M. N. (2004). Phenyl substitution of furamidine markedly potentiates its antiparasitic activity against Trypanosoma cruzi and Leishmania amazonensis. Biochemical Pharmacology 68, 593-600, doi:10.1016/j.bcp.2004.04.019 [doi];S0006295204003089 [pii].

de Souza, E. M., Menna-Barreto, R., Araujo-Jorge, T. C., Kumar, A., Hu, Q., Boykin, D. W. and Soeiro, M. N. (2006). Antiparasitic activity of aromatic diamidines is related to apoptosis-like death in Trypanosoma cruzi. Parasitology 133, 75-79, doi:S0031182006000084 [pii];10.1017/ S0031182006000084 [doi].

Elsheikha, H. M. and Mansfield, L. S. (2004). Determination of the activity of sulfadiazine against Besnoitia darlingi tachyzoites in cultured cells. Parasitology Research 93, 423-426, doi:10.1007/s00436-004-1133-5 [doi].

Esposito, M., Moores, S., Naguleswaran, A., Muller, J. and Hemphill, A. (2007a). Induction of tachyzoite egress from cells infected with the protozoan Neospora caninum by nitro- and bromo-thiazolides, a class of broad-spectrum anti-parasitic drugs. International fournal for Parasitology 37, 1143-1152.

Esposito, M., Muller, N. and Hemphill, A. (2007b). Structure-activity relationships from in vitro efficacies of the thiazolide series against the intracellular apicomplexan protozoan Neospora caninum. International Fournal for Parasitology 37, 183-190.

Esposito, M., Stettler, R., Moores, S. L., Pidathala, C., Muller, N., Stachulski, A., Berry, N. G., Rossignol, J. F. and Hemphill, A. (2005). In vitro efficacies of nitazoxanide and other thiazolides against Neospora caninum tachyzoites reveal antiparasitic activity independent of the nitro group. Antimicrobial Agents and Chemotherapy 49, 3715-3723.

Fernandez-Garcia, A., Alvarez-Garcia, G., Risco-Castillo, V., Aguado-Martinez, A., Marcen, J. M., Rojo-Montejo, S., Castillo, J. A. and Ortega-Mora, L. M. (2010). Development and use of an indirect ELISA in an outbreak of bovine besnoitiosis in Spain. Veterinary Record 166, 818-822, doi:166/26/818 [pii];10.1136/vr.b4874 [doi].

Gollnick, N. S., Gentile, A. and Schares, G. (2010). Diagnosis of bovine besnoitiosis in a bull born in Italy. Veterinary Record 166, 599, doi:166/19/ 599 [pii];10.1136/vr.c2314 [doi].

Gomez, C., Esther, R.M., Calixto-Galvez, M., Medel, O. and Rodriguez, M.A. (2010). Regulation of gene expression in protozoa parasites. Fournal of Biomedical Biotechnology 2010, 726045, doi:10.1155/ 2010/726045.

Gondim, L. F. (2006). Neospora caninum in wildlife. Trends in Parasitology 22, 247-252

Hemphill, A., Vonlaufen, N., Naguleswaran, A., Keller, N., Riesen, M., Guetg, N., Srinivasan, S. and Alaeddine, F. (2004). Tissue culture and explant approaches to studying and visualizing Neospora caninum and its interactions with the host cell. Microscopy and Microanalysis 10, 602-620.

Jacquiet, P., Lienard, E. and Franc, M. (2010). Bovine besnoitiosis: Epidemiological and clinical aspects. Veterinary Parasitology, doi:S03044017(10)00454-1 [pii];10.1016/j.vetpar.2010.08.013 [doi].

Juste, R. A., Cuervo, L. A., Marco, J. C. and Oregui, L. M. (1990). La besnoitiosis bovina: desconocida en España? Medicina Veterinária 7, 613-618.

Kim, J. T., Park, J. Y., Seo, H. S., Oh, H. G., Noh, J. W., Kim, J. H., Kim, D. Y. and Youn, H. J. (2002). In vitro antiprotozoal effects of artemisinin on Neospora caninum. Veterinary Parasitology 103, 53-63. 
Kwon, H. J., Kim, J.H., Kim, M., Lee, J. K., Hwang, W. S. and Kim, D.Y. (2003). Anti-parasitic activity of depudecin on Neospora caninum via the inhibition of histone deacetylase. Veterinary Parasitology 112, 269-276

Leepin, A., Studli, A., Brun, R., Stephens, C. E., Boykin, D. W. and Hemphill, A. (2008). Host cells participate in the in vitro effects of nove diamidine analogues against tachyzoites of the intracellular apicomplexan parasites Neospora caninum and Toxoplasma gondii. Antimicrobial Agent and Chemotherapy 52, 1999-2008, doi:AAC.01236-07 [pii];10.1128/ AAC.01236-07 [doi]

Lindsay, D. S. and Dubey, J. P. (1989). Evaluation of anti-coccidial drugs' inhibition of Neospora caninum development in cell cultures. Fournal of Parasitology 75, 990-992.

Lindsay, D. S., Rippey, N. S., Cole, R. A., Parsons, L. C., Dubey, J. P. Tidwell, R. R. and Blagburn, B. L. (1994). Examination of the activities of 43 chemotherapeutic agents against Neospora caninum tachyzoites in cultured cells. American Fournal of Veterinary Research 55, 976-981.

Pols, J. W. (1960). Studies on Bovine besnoitiosis with special reference to the aetiology. Onderstepoort Fournal of Veterinary Research 28, 265-356.

Schares, G., Basso, W., Majzoub, M., Cortes, H. C., Rostaher, A., Selmair, J., Hermanns, W., Conraths, F. J. and Gollnick, N. S. (2009)
First in vitro isolation of Besnoitia besnoiti from chronically infected cattle in Germany. Veterinary Parasitology 163, 315-322, doi:S0304-4017(09) 00276-3 [pii];10.1016/j.vetpar.2009.04.033 [doi]

Shkap, V., de Waal, D. T. and Potgieter, F. T. (1985). Chemotherapy of experimental Besnoitia besnoiti infection in rabbits. Onderstepoort Fournal of Veterinary Research 52, 289.

Shkap, V., Pipano, E. and Ungar-Waron, H. (1987). Besnoitia besnoiti: chemotherapeutic trials in vivo and in vitro. Revue d'Elevage et de Medecine Veterinaire des Pays Tropicaux 40, 259-264.

Wang, M. Z., Zhu, X., Srivastava, A., Liu, Q., Sweat, J. M., Pandharkar, T., Stephens, C. E., Riccio, E., Parman, T., Munde, M., Mandal, S., Madhubala, R., Tidwell, R. R., Wilson, W.D. Boykin, D. W., Hall, J. E., Kyle, D. E. and Werbovetz, K. A. (2010)

Novel arylimidamides for treatment of visceral leishmaniasis. Antimicrobial Agents and Chemotherapy 54, 2507-2516, doi:AAC.00250-10 [pii];10.1128/ AAC.00250-10 [doi].

Youn, H. J., Lakritz, J., Rottinghaus, G. E., Seo, H.S., Kim, D. Y., Cho, M. H. and Marsh, A.E. (2004). Anti-protozoal efficacy of high performance liquid chromatography fractions of Torilis japonica and Sophora flavescens extracts on Neospora caninum and Toxoplasma gondii. Veterinary Parasitology 125, 409-414. 